\title{
Quelques aspects de l'œuvre de Jean-Marie Legay
}

\author{
Franck Varenne \\ Philosophe, Université de Rouen, UFR LSH, Département de philosophie, 76821 Mont-Saint-Aignan, France
}

Quand on essaie aujourd'hui de retracer l'origine des techniques contemporaines de modélisation et de simulation du développement et de la croissance des plantes, on tombe rapidement sur plusieurs travaux importants de Jean-Marie Legay ${ }^{1}$. Un de ces travaux concerne la modélisation de la ramification de certaines plantes végétatives dont le gui. Il a été publié en 1971 dans le journal fondé par Nicholas Rashevsky, le Bulletin of Mathematical Biophysics $(B M P)$, sous le titre «Contribution à l'étude de la forme des plantes: discussion d'un modèle de ramification $^{2} »$. Ce travail se caractérise par le fait qu'il est un des premiers à l'époque à mettre systématiquement en regard plusieurs approches distinctes de modélisation formelle de ce qui commençait - avec les travaux contemporains des botanistes Nozeran, Hallé et Oldeman - à être nommé « architecture » et «modèle architectural » des plantes. Legay y compare les mérites de l'approche métabolique (inspirée alors de la physiologie générale et de la biophysique théorique de Nicholas Rashevsky) avec ceux de l'approche populationnelle (inspirée par la dynamique des populations de cellules) d'origine, quant à elle, à la fois biométrique et de dynamique des populations. On y voit un auteur à l'aise tout autant dans les conceptualisations et les mathématisations abstraites de la biophysique que dans les techniques statistiques issues de la biométrie au sens strict (statistique).

Trois ans plus tôt, en 1968, Rashevsky avait déjà été très intéressé par les premières propositions de Legay. Son journal avait ainsi précédemment publié un premier

\footnotetext{
Auteur correspondant : franck.varenne@univ-rouen.fr

1 Voir aussi, dans ce numéro et le suivant, les autres hommages à Jean-Marie Legay.

2 Legay, J.-M., 1971. Contribution à l'étude de la forme des plantes : discussion d'un modèle de ramification, $B M P, 33,3$, 387-401.
}

article de Legay intitulé «Éléments d'une théorie générale de la croissance d'une population ${ }^{3}$ ». Legay y introduisait une grammaire formelle des filiations cellulaires, l'année même où paraissaient - dans le Journal of Theoretical Biology - les deux articles d'Aristid Lindenmayer qui donneront naissance à la grammaire formelle à réécriture nommée par la suite L-système. Cette grammaire de Legay était également un langage formel récursif. Un tel langage se donne 1) un alphabet de base, c'est-à-dire une liste de symboles élémentaires recevables dans ce langage, 2) des règles de combinaisons recevables de ces symboles élémentaires (règles de formation ou règles syntaxiques des expressions complexes qui sont dites alors «bien formées »), 3) des axiomes, c'est-à-dire un certain nombre d'expressions bien formées que l'on postule comme valides, 4) des règles de transformation de formes d'expressions bien formées en d'autres formes d'expressions bien formées, 5) une possibilité d'itérer un nombre quelconque (fini) de fois ces règles pour aboutir à des expressions bien formées nouvelles. Ce sont les règles de transformation qui essentiellement donnent sa dynamique déductive au langage et l'instituent comme calcul. En s'installant du point de vue des unités biologiques discrètes, ce genre de calcul peut aussi bien simuler pas à pas un processus biologique de développement - l'apparition de nouvelles cellules au niveau cellulaire (Lindenmayer) ou de nouvelles unités de croissance au niveau organique (Prusinkiewicz ${ }^{4}$ ) - qu'un processus de filiation entre cellules ou entre individus (Legay). Or, à la différence de celui de Lindenmayer, le langage formel de Legay faisait d'emblée une place considérable aux probabilités de ramification et de différenciation (l'application

\footnotetext{
Legay, J.-M., 1968. Éléments d'une théorie générale de la croissance d'une population, $B M P, 30,33-46$.

4 Prusinkiewicz, P., Lindenmayer, A., 1990. The Algorithmic Beauty of Plants, New York, Springer Verlag.
} 
des règles de transformation était soumise à des lois probabilistes) alors que celui de Lindenmayer mettait en œuvre uniquement une théorie déterministe, il est vrai spatialisée, ce qui le rapprochait davantage des automates cellulaires développés par ailleurs en informatique de simulation. Legay anticipait, de ce point de vue, sur les formes de L-systèmes assouplies et sensibles à l'environnement qu'on appellera les L-systèmes paramétriques ${ }^{5}$.

Ces quelques points d'histoire des sciences permettent d'entrevoir dans quelle mesure et pourquoi, dès la fin des années 1960, Legay ne dépendait pas d'une chapelle et était l'un des premiers à posséder une réelle hauteur de vue sur la diversité des techniques de modélisation et de théorisation des êtres vivants. Même s'il fut d'abord formé à la biométrie et aux études biologiques de terrain, Legay ne rejetait pas toute entreprise de théorisation mathématique en biologie, au contraire. Au fond, il acceptaitl'approche théorique dans un esprit de curiosité scientifique mais aussi de tolérance et d'égalité avec d'autres approches plus directement issues des mesures de terrain. Toutefois, il lui imposait bien sûr l'épreuve expérimentale (ce dont il discutait souvent avec Rashevsky) mais aussi, ce qui était moins fréquent, la confrontation latérale avec les autres techniques de modélisation : ainsi s'explique la structure comparativiste $^{6}$ de son article de 1971. Assez étonnamment pour l'époque, l'ingénieur agronome acceptait donc les hypothèses théoriques. Il acceptait de les considérer à la manière même dont il avait pris au sérieux - à égalité avec des savoirs déjà mieux formalisés - certaines pratiques très concrètes et empiriques, certains savoir-faire millénaires au sujet du ver à soie, notamment, lorsqu'il s'était agi pour lui - dans l'Inra des années 1950 et de la reconstruction -, de rendre plus systématique et plus rigoureux cet ensemble de savoirfaire traditionnels. Le ver à soie fut en effet son premier objet biologique. Il fut d'abord pour lui un «modèle

\footnotetext{
5 C'est-à-dire quel'applicabilité des règles de transformation du langage formel simulant, par exemple, la décision de ramifier pour un bourgeon est soumise à un état de l'environnement de l'organisme à un moment donné, à un endroit donné, et pas seulement à une loi probabiliste : on ajoute des paramètres dont les valeurs changent au cours du développement. L'applicabilité des règles de transformation change donc aussi au cours de l'histoire de la croissance ou du développement. Cf. Kurth, W., 1995. Stochastic sensitive growth grammars: A basis for morphological models of tree growth. Communication au colloque L'arbre-Biologie et développement, Montpellier, 11-16 septembre, 15 pages, accessible sur htttp://www.uni-forst.gwdg.de/ $\sim$ wkurth/. Cf. aussi la présentation et l'analyse épistémologique comparative dans Varenne, F., 2007. Du modèle à la simulation informatique, Paris, Vrin, 160-163.

6 Legay y compare des modèles de croissance inspirés aussi bien de la biophysique théorique que de la biométrie. $C f$. Varenne, F., 2010. Formaliser le vivant: lois, théories, modèles? Paris, Hermann, 303-315.
}

expérimental » au sens classique qu'a cette expression en médecine et biologie, comme l'est la drosophile ou la bactérie Escherichia coli. Mais la culture du ver à soie présente bien d'autres aspects - agronomiques, sociologiques et économiques - dont l'ingénieur de l'Inra doit également prendre la mesure. À le pratiquer, cet objet biologique se révéla donc très pluri-aspectuel: dans un entretien remontant à 2001 (non publié), Legay m'avait indiqué que ces travaux tous azimuts sur le ver à soie furent en grande partie ce qui l'incita de manière précoce à prôner une approche de tolérance, que l'on dit aujourd'hui «pluridisciplinaire » et qu'il dira lui-même plus tard - en manière de boutade - « indisciplinée ». C'est aussi ce qui l'amène dès 1971 à considérer que tous les modèles ne sont que des outils en vue d'un certain objectif, objectif que le modélisateur doit avant toutes choses définir et expliciter. Même si un modèle se révèle être une représentation - partielle et aspectuelle -, sa fonction ne peut jamais consister en ce seul fait de représenter.

La perspicacité de Legay se remarque aussi sur un autre front : celui qui touche à la réflexion sur l'informatisation massive des techniques de modélisation dans les quarante dernières années. Au début des années 1970, alors qu'il fait ses premiers pas à la DGRST, il est cofondateur de l'association Informatique et Biosphère, sorte de société savante, promotrice de l'informatique dans les milieux des sciences de l'environnement. Cette association éphémère créera une revue, Informatique et Biosphère, elle-même éphémère, mais qui publiera le premier long manifeste de Legay ${ }^{7}$ en faveur de la « méthode des modèles » et qui sera doublée d'une présentation de l'interprétation non représentationnaliste des modèles déjà rôdée dans l'article de 1971. Cet article long sera souvent cité dans la littérature francophone de modélisation avant qu'il ne soit détrôné par l'ouvrage de 1997, L'Expérience et le Modèle ${ }^{8}$. Dès 1973, comme praticien averti, Legay y entre dans le détail des analyses épistémologiques concernant l'usage des modèles en science et argumente en faveur de la thèse du modèle outil. Par là, Legay fait son entrée dans la réflexion épistémologique en interpellant les philosophes eux-mêmes, sur leur terrain.

Legay reconnaîtra plus tard que la vision de l'informatique portée par l'association Informatique et Biosphère était étriquée et sur le point d'être déjà dépassée, même à l'époque, ce qui explique en partie sa disparition rapide. C'est que l'informatique y était vue seulement sous l'angle informationnel, comme un instrument, certes interdisciplinaire par nature, mais servant seulement au stockage des données, à leur classement, à leur traitement statistique et à leur communication. Legay

\footnotetext{
Legay, J.-M., 1973. La méthode des modèles, état actuel de la méthode expérimentale, Informatique et Biosphère, 1, 5-73.

8 Legay, J.-M., 1997. L'Expérience et le Modèle. Un discours sur la méthode, collection Sciences en questions, Inra Éditions.
} 
admettra que c'était occulter l'essor contemporain de ce qu'il appellera l'informatique " agissante ». Dans cette informatique nouvelle, le programme ne sert plus à la programmation d'un calcul statistique de données mais à la programmation de certaines idées. C'est une programmation agissante au sens où elle n'est pas au service $d^{\prime}$ un modèle mathématique préalable mais qu'elle permet de procéder - de manière active - au test direct de certaines idées. Toutefois, même dans ce cas de figure, notamment lorsqu'il conduit à une simulation informatique de plus en plus réaliste ou, plus exactement, de plus en plus intégratrice de données disparates, le modèle de simulation n'est pas une représentation mais seulement un outil. À ce titre, il ne peut être comparé avec une expérience puisque l'expérience relève toujours d'une interaction globale entre le modèle agissant et le terrain.

Legay, je crois, serait d'accord pour dire qu'un modélisateur, l'ordinateur et le terrain qu'il simule forment à eux trois un système complexe. Legay définissait un système complexe par une double négation : comme un système qui change de nature (n'a donc plus sa nature initiale et n'est donc plus complexe) quand on lui retire un élément, c'est-à-dire qu'il ne possède plus un élément donné qui n'était pas plus important que tous les autres mais qui se trouvait interagir avec tous les autres. Par exemple, un champ délaissé qui n'est plus cultivé par un agriculteur (le système a perdu cet élément « agriculteur ») déploie une trajectoire beaucoup plus prévisible et moins complexe en ce sens que si on étudie le système « champ/agriculteur » et son devenir. Ainsi peut-on trouver pour finir une cohérence entre sa thèse épistémologique sur les modèles et sa caractérisation des systèmes complexes : une simulation informatique, seule, n'est pas une expérience au sens fort car, en l'isolant, on perd la dimension complexe de toute entreprise d'expérimentation scientifique dès lors qu'il y manque le modélisateur, le terrain d'inspiration originelle ainsi que les objectifs de simulation, qui en interagissant à eux trois forment bien un système complexe. Vouloir prendre une simulation séparée de son modélisateur et de son ancrage empirique originel pour une expérience au sens fort, vouloir la considérer à elle seule comme une captation intégrale, dans la machine, de la complexité des systèmes étudiés par ailleurs en sciences de la vie et de l'environnement, ce serait croire que l'aventure scientifique elle-même n'aurait plus désormais, avec l'essor contemporain de l'informatique de modélisation, à être considérée elle-même comme complexe. Malgré toute son indiscipline militante et tout son esprit d'ouverture et de tolérance, c'est bien cette seule idée au fond qui lui paraissait déraisonnable. À juste titre. 